\title{
Biologia populacional de Corydoras flaveolus (Siluriformes, Callichthyidae) no Rio Passa Cinco, sub-bacia do Rio Corumbataí, São Paulo, Brasil
}

\author{
Giulianna Rodrigues Rondineli ${ }^{1,2}$ \& Francisco Manoel de Souza Braga ${ }^{1}$ \\ ${ }^{1}$ Departamento de Zoologia, Instituto de Biociências, Universidade Estadual Paulista - UNESP, \\ Av. 24A, no. 1515, Bela Vista, CP 199, CEP 13506-900, Rio Claro, SP, Brazil \\ ${ }^{2}$ Autor para correspondência: Giulianna Rodrigues Rondineli, e-mail: giulianna.rondineli@gmail.com
}

RONDINELI, G.R. \& BRAGA, F.M.S. Population biology of Corydoras flaveolus (Siluriformes, Callichthyidae) in Passa Cinco stream, Corumbatai river sub-basin, São Paulo state, Brazil. Biota Neotrop., 9(4): http:// www.biotaneotropica.org.br/v9n4/en/abstract?article+bn00809042009

\begin{abstract}
The aim of this work was to characterize the population of Corydoras flaveolus in Passa Cinco stream, regarding length structure, sex ratio and reproductive aspects, in accordance with drought and rainy season periods, as well as to analyze its corporal condition. Six samplings were accomplished in five different points of Passa Cinco stream (contemplating sites of orders two to six) through use of a sieve, electric fishery equipment and fish-traps. It was captured 70 individuals, composed by 35 males and 35 females. There was not significant variation in repletion degree and accumulated fat in visceral cavity of the individuals analyzed during the considered periods. The reproductive period of $C$. flaveolus was extensive and more intense in the rainy season due to the largest percentage of individuals with mature gonads and higher value of gonadossomatic relationship founded in that period. The average of absolute fecundity was of 137 oocytes, and the intermittent type of spawning was performed. The females were larger and heavier than males in the same lenght. There was no difference in corporal condition of both sexes between the periods considered.
\end{abstract}

Keywords: population structure, reproduction, fecundity, corporal condition.

RONDINELI, G.R. \& BRAGA, F.M.S. Biologia populacional de Corydoras flaveolus (Siluriformes, Callichthyidae) no Rio Passa Cinco, sub-bacia do Rio Corumbataí, São Paulo, Brasil. Biota Neotrop., 9(4): http://www.biotaneotropica.org.br/v9n4/pt/abstract?article+bn00809042009

Resumo: O objetivo desse trabalho foi caracterizar a população de Corydoras flaveolus do Rio Passa Cinco com relação à estrutura em comprimento, proporção sexual e aspectos reprodutivos de acordo com os períodos de seca e cheia, bem como analisar sua condição corporal. Seis amostragens foram realizadas em cinco pontos distintos no Rio Passa Cinco (contemplando trechos de ordem dois, três, quatro, cinco e seis) com o uso de peneira, pesca elétrica e covos. Foram capturados 70 indivíduos, sendo 35 machos e 35 fêmeas. Não houve variação significativa nos graus de repleção e de gordura acumulada na cavidade visceral dos exemplares analisados entre os períodos considerados. O período reprodutivo de $C$. flaveolus foi extenso, sendo mais intenso na cheia, visto a maior porcentagem de indivíduos com gônadas maduras e o maior valor da relação gonadossomática durante esse período. A fecundidade absoluta média foi de 137 ovócitos, sendo a desova do tipo intermitente. As fêmeas foram maiores e relativamente mais pesadas do que os machos. Não houve diferença significativa na condição corporal de ambos os sexos entre os períodos considerados.

Palavras-chave: estrutura populacional, reprodução, fecundidade, condição corporal. 


\section{Introdução}

O gênero Corydoras Lacepède, 1803 é composto por 143 espécies (Reis 2003) amplamente distribuídas na América do Sul, ocorrendo em uma variedade de hábitats (Britto et al. 2007). De acordo com Reis (2003), em média, duas novas espécies de Corydoras têm sido descritas todo o ano durante as últimas décadas, o que se confirma com as descrições de Corydoras difluviatilis Britto \& Castro (2002), Corydoras diphyes Axenrot \& Kullander (2003), Corydoras tukano Britto \& Lima (2003) e Corydoras ortegai Britto, Lima \& Hidalgo (2007).

Existe, na literatura, uma série de informações sobre espécies de Corydoras, talvez porque muitas delas são de interesse em aquariofilia. Aspectos biológicos, principalmente reprodutivos, estão presentes nos trabalhos de Loir et al. (1989), Aranha et al. (1993), Bervian \& Fontoura (1994), Paxton (1997), Kohda et al. (1995), Pruzsinszky \& Ladich (1998), Kaatz \& Lobel (1999), Alkins-Koo (2000), Kohda et al. (2002), Huysentruyt \& Adriaens (2005) e Mazzoldi et al. (2007). Estudos citogenéticos foram conduzidos por Shimabukuro-Dias et al. (2004) e Artoni et al. (2006). E a variação geográfica foi analisada por Shibatta \& Hoffmann (2005).

Dentre os trabalhos revisados, apenas o de Strauss (1985), que apresenta uma análise de caracteres merísticos e morfométricos de 123 espécies e subespécies de Corydoras, o de Britto (2003), que apresenta uma hipótese sobre as relações filogenéticas da subfamília Corydoradinae, e o de Spadella et al. (2007), que realiza uma análise comparativa da espermiogênese em Callichthyidae, remetem-se à espécie foco do presente estudo.

Corydoras flaveolus Ihering, 1911 é uma espécie de pequeno porte e sua distribuição geográfica compreende a bacia do Alto Rio Paraná (Reis 2003). No Rio Passa Cinco, sub-bacia do Rio Corumbataí, C. flaveolus é uma das espécies mais abundantes (Rondineli, G.R. \& Braga, F.M.S. dados não publicados).

O objetivo desse trabalho foi caracterizar a população de Corydoras flaveolus do Rio Passa Cinco com relação à estrutura em comprimento, proporção sexual e aspectos reprodutivos de acordo com os períodos de seca e cheia, bem como analisar sua condição corporal, contribuindo, assim, com informações sobre a biologia dessa espécie.

\section{Material e Métodos}

O presente estudo foi realizado no Rio Passa Cinco, um dos principais rios da sub-bacia do Rio Corumbataí, que nasce na serra da Cachoeira, pertencente ao complexo da serra de Itaqueri, município de Itirapina, e apresenta uma área de drenagem com $525 \mathrm{~km}^{2}$, percorrendo cerca de $60 \mathrm{~km}$ desde suas nascentes, com altitude de $1000 \mathrm{~m}$, até sua foz no Rio Corumbataí, com altitude de $480 \mathrm{~m}$ (Garcia et al. 2004). Atualmente, possui $51,72 \%$ da sua superfície ocupada por áreas de pastagem, $14,13 \%$ por cana-de-açúcar, $15,67 \%$ por floresta nativa e $0,74 \%$ por cerrado (Valente \& Vettorazzi 2002).

Foram realizadas seis amostragens em cinco pontos distintos no Rio Passa Cinco, contemplando trechos de ordem dois, três, quatro, cinco e seis, segundo a classificação de Strahler (1952), com as seguintes coordenadas geográficas: ponto 1 (ordem 2$)-22^{\circ} 23^{\prime} 36^{\prime \prime} \mathrm{S}$ $47^{\circ} 53^{\prime} 08^{\prime \prime} \mathrm{O}$, ponto 2 (ordem 3 ) - $22^{\circ} 22^{\prime} 10^{\prime \prime} \mathrm{S} 47^{\circ} 51^{\prime} 22^{\prime \prime} \mathrm{O}$, ponto 3 (ordem 4 ) $-22^{\circ} 21^{\prime} 63^{\prime \prime} \mathrm{S} 47^{\circ} 48^{\prime} 48^{\prime \prime} \mathrm{O}$, ponto 4 (ordem 5) $-22^{\circ} 24^{\prime} 74^{\prime \prime} \mathrm{S} 47^{\circ} 43^{\prime} 34^{\prime \prime} \mathrm{O}$ e ponto 5 (ordem 6) - $22^{\circ} 30^{\prime} 97^{\prime \prime} \mathrm{S}$ 47 39' 49" O nos meses de maio, julho, setembro e novembro de 2005 e janeiro e março de 2006 (Figura 1).

Os pontos amostrais foram caracterizados de acordo com o tipo de substrato predominante (matacão, seixo e areia), vegetação marginal (presença ou ausência), grau de sombreamento (levando em consideração a presença de cobertura vegetal e fazendo uso de uma escala arbitrária de quatro classes: 0-25\%, 26-50\%, 51-75\% e $>76 \%$ ), tipo de corrente (presença de rio corrente, corredeira e/ou poção) e profundidade média (em metros).

Os dados de pluviosidade e temperatura do ar para o período de estudo foram obtidas junto ao CEAPLA (Centro de Análise e Planejamento Ambiental), da Universidade Estadual Paulista, em Rio Claro, sendo estas informações fundamentais para a definição das estações seca e chuvosa.

Os apetrechos de pesca utilizados foram: peneira, pesca elétrica e covos. A peneira foi passada na vegetação marginal por dez vezes em cada um dos pontos (exceto no ponto de ordem 2 devido às características do local: ausência de vegetação e pequeno volume de água). $\mathrm{O}$ aparelho de pesca elétrica (que consiste em um gerador que fornece energia - $110 \mathrm{~V}$ - para um retificador de corrente que tem a capacidade máxima de aumentar a voltagem - para até $1500 \mathrm{~V}$ - e reduzir a amperagem - para 2 A) foi utilizado nos três primeiros pontos (pontos 1, 2 e 3). Nesses locais foram escolhidos trechos de $50 \mathrm{~m}$, onde o aparelho foi passado por uma única vez no sentido jusante-montante, não utilizando rede de contenção. Dois covos de $50 \mathrm{~cm}$ de comprimento e $20 \mathrm{~cm}$ de diâmetro foram utilizados em todos os pontos e dentro de cada covo utilizou-se como isca porções de ração animal.

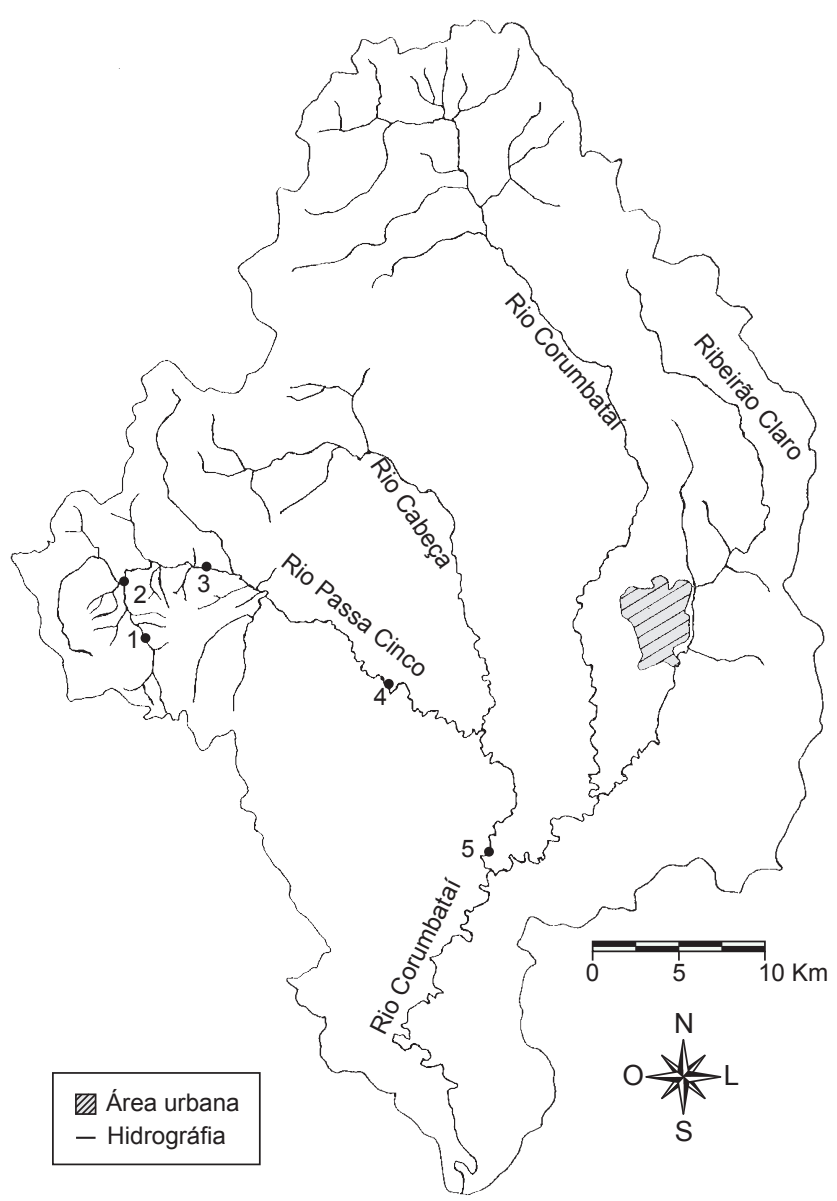

Figura 1. Mapa hidrológico da sub-bacia do rio Corumbataí, com destaque para os pontos amostrais (modificado de Garcia et al. 2004). (1- Ponto 1; 2 - Ponto 2; 3 - Ponto 3; 4 - Ponto 4; e 5 - Ponto 5).

Figure 1. Hydrographic map of the Corumbataí river sub-basin, showing the sampled sites (modified from Garcia et al., 2004). (1 - Sample site 1;2-Sample site 2; 3 - Sample site 3; 4 - Sample site 4; and 5 - Sample site 5). 
Após cada uma das coletas, os peixes foram colocados em sacos plásticos, fixados em formalina 10\% durante 2 dias e posteriormente transferidos para álcool $70 \%$ até a realização das análises.

No laboratório, os exemplares de Corydoras flaveolus foram separados das demais espécies e tomados os seguintes dados: comprimento total e padrão em centímetros, peso total em gramas, grau de repleção do estômago (GR), grau de gordura acumulada na cavidade visceral (GA), sexo e estádio de maturação gonadal (Braga 1990). Os espécimes foram depositados no Laboratório de Ictiologia, no Departamento de Zoologia da Universidade Estadual Paulista, campus de Rio Claro.

Ao grau de repleção estomacal (estado de enchimento do estômago) foram atribuídos os seguintes valores: 1 para estômagos considerados vazios, 2 para estômagos parcialmente cheios e 3 para estômagos completamente cheios (Braga 1990).

Para o grau de gordura acumulada na cavidade visceral foram atribuídas três categorias: 1 para a cavidade visceral que não apresenta gordura, 2 para a cavidade visceral parcialmente preenchida com gordura e 3 para a cavidade visceral repleta de gordura (Braga 1990).

De acordo com uma escala previamente estabelecida (Vazzoler 1996), quatro diferentes estádios de maturação gonadal foram considerados: A para gônadas imaturas, B para gônadas em maturação ou em repouso, $\mathrm{C}$ para gônadas maduras e $\mathrm{D}$ para aquelas consideradas esgotadas.

Para o estudo da fecundidade, os ovários em estádio C de maturação foram retirados da cavidade visceral, pesados e imersos em solução de Gilson (Vazzoler 1996).

A estrutura populacional de Corydoras flaveolus foi analisada com relação à proporção sexual (Vazzoler 1996) e à distribuição de comprimento por sexo; o teste de Wilcoxon (Sokal \& Rohlf 1995) foi utilizado para constatar se as distribuições de comprimento entre os sexos apresentavam diferenças, sendo 0,05 o nível de significância considerado.

Para testar a hipótese de que os graus de repleção e gordura acumulada na cavidade visceral dependem da época do ano não levando em consideração o sexo, foi aplicado um teste de $\chi^{2}$ para testar a hipótese de que duas variáveis categorizadas são independentes (Vieira 2004), sendo 0,05 o nível de significância considerado.

Explanações sobre o período reprodutivo da espécie foram feitas através das frequiências de indivíduos com gônadas nos diferentes estádios de maturidade e a variação temporal na relação gonadossomática (RGS), que expressa a porcentagem que as gônadas representam do peso total dos indivíduos (Vazzoler 1996).

Os ovários após permanecerem em solução de Gilson, para dissociação total dos ovócitos do epitélio germinativo e folículos ovarianos, foram lavados em sucessivos banhos em álcool a 70\%, limpos do epitélio ovariano e estocados em álcool a $70 \%$.

Dos ovários dissociados, ovócitos vitelogênicos foram contados em sua totalidade e medidos sob estereomicroscópio aleatoriamente, utilizando-se de uma ocular micrométrica $(1 \mathrm{~d}$. o. m. $=61,2 \mu \mathrm{m})$. Uma vez conhecido o número de ovócitos contido nos ovários e conhecendo-se a distribuição dos diâmetros de ovócitos, estimou-se a fecundidade absoluta para cada fêmea.

A fecundidade da população foi equacionada correlacionando-se as fecundidades absolutas com os pesos totais das fêmeas, segundo a expressão $\mathrm{F}=\mathrm{A}+\mathrm{BPt}$, sendo $\mathrm{F}$ a fecundidade, $\mathrm{Pt}$ o peso total, $\mathrm{A}$ o intercepto e B o coeficiente angular, estimados pelo método dos mínimos quadrados (Sparre \& Venema 1997).

Através da distribuição de frequiência dos diâmetros dos ovócitos, verificou-se o tipo de desova pela presença de uma moda de ovócitos vitelogênicos (desova total) ou mais de uma moda de ovócitos vitelogênicos (desova parcelada), segundo Vazzoler (1996).
Um teste $\mathrm{t}$ foi aplicado às relações peso e comprimento de machos e fêmeas de modo a verificar possíveis diferenças entre as retas de regressão (Fowler et al. 1998).

Para avaliar a condição corporal da espécie, independente do sexo, foi aplicada uma análise de covariância (Cetra 2005) cujo modelo usado foi: $P=C t e+C+E+C * \mathrm{E}+$ erro; onde $\mathrm{P}$ representa o peso dos indivíduos (g), Cte é a constante do modelo, C é o comprimento padrão (mm), E é a época (seca ou cheia) e erro corresponde ao resíduo. Aos dados de peso e comprimento foi aplicada uma transformação logarítmica $(\ln +1)$ e a distribuição dos resíduos foi verificada através do teste de Lilliefors.

\section{Resultados}

Durante o período de estudo, as menores temperaturas do ar foram registradas no mês de julho de $2005\left(18,85^{\circ} \mathrm{C}\right)$ e as maiores no mês de janeiro de $2006\left(25,92^{\circ} \mathrm{C}\right)$. A pluviosidade total variou de 3,6 mm em julho de 2005 a $175,5 \mathrm{~mm}$ em dezembro de 2005 . Na Figura 2 estão apresentados os valores médios mensais da temperatura do ar e medianos da pluviosidade. Com isso, verificou-se que as três primeiras amostras pertenceram à estação de seca (outono/inverno) e as três últimas à estação de cheia (primavera/verão).

O tipo de substrato, grau de sombreamento, tipo de corrente, vegetação marginal e profundidade média encontrados para cada um dos pontos amostrais encontram-se na Tabela 1. O tipo de substrato mudou gradualmente do ponto 1 ao ponto 5 , com a predominância de matacão/seixo nos pontos localizados mais à montante (1 e 2) e areia nos pontos mais à jusante (4 e 5). No ponto 3 (um local de transição cabeceira-foz), matacão, seixo e areia estiveram presentes de modo equilibrado. A vegetação marginal (presença de gramíneas ao longo das margens do riacho) ocorreu em todos os pontos amostrais, com exceção do ponto 1 . O grau de sombreamento diminui da cabeceira à foz. Os tipos de corrente predominantes foram: corredeira no ponto 1 ; corredeira e rio corrente no ponto 2 ; corredeira, rio corrente e poço no ponto 3; e rio corrente nos pontos 4 e 5 . A profundidade média aumentou no sentido montante-jusante.

Foram capturados 70 indivíduos de Corydoras flaveolus no Rio Passa Cinco, com as seguintes capturas por ponto (em número de indivíduos): ponto $1=0$, ponto $2=22$, ponto $3=31$, ponto $4=5 \mathrm{e}$ ponto $5=12$.

Dos 70 indivíduos capturados, 35 eram machos e 35 fêmeas, não apresentando, portanto, proporção sexual diferente do esperado (1:1). O menor indivíduo capturado, um macho, apresentou $23 \mathrm{~mm}$ de comprimento padrão e o maior, uma fêmea, 39 mm (Figura 3). Os resultados do teste de Wilcoxon indicaram diferenças significativas

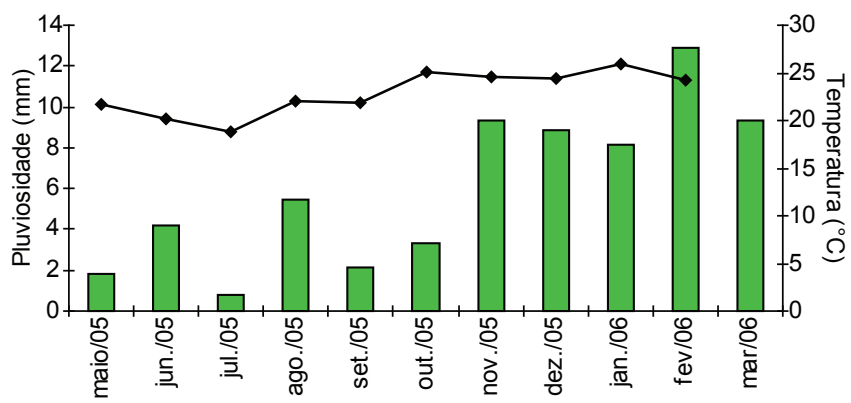

Figura 2. Distribuição dos valores médios mensais de temperatura do ar e valores medianos da pluviosidade mensal no período de amostragem (maio de 2005 a março de 2006).

Figure 2. Distribution of monthly medium values of air temperature and monthly median values of rainfall level in the sampling period (May of 2005 to March of 2006). 
Tabela 1. Tipo de substrato, vegetação marginal, grau de sombreamento, tipo de corrente e profundidade média encontrados em cada um dos pontos amostrais.

Table 1. Bottom type, marginal vegetation, degree of shading, current type and mean width founded in each of the sampling sites.

\begin{tabular}{cllclr}
\hline $\begin{array}{c}\text { Pontos } \\
\text { amostrais }\end{array}$ & \multicolumn{1}{c}{$\begin{array}{c}\text { Tipo de } \\
\text { substrato }\end{array}$} & $\begin{array}{c}\text { Vegetação } \\
\text { marginal }\end{array}$ & $\begin{array}{c}\text { Grau de } \\
\text { sombreamento }\end{array}$ & $\begin{array}{c}\text { Tipo de } \\
\text { corrente }\end{array}$ & $\begin{array}{c}\text { Profundidade } \\
\text { média (m) }\end{array}$ \\
\hline 1 & Matacão & ausente & $>76 \%$ & corredeira & 2,3 \\
2 & matacão, seixo & presente & $51-75 \%$ & corredeira, rio corrente & 5,8 \\
3 & matacão, seixo, areia & presente & $26-50 \%$ & corredeira, rio corrente, poço & 8,9 \\
4 & Areia & presente & $26-50 \%$ & rio corrente & 10,8 \\
5 & Areia & presente & $0-25 \%$ & rio corrente & 15,3 \\
\hline
\end{tabular}

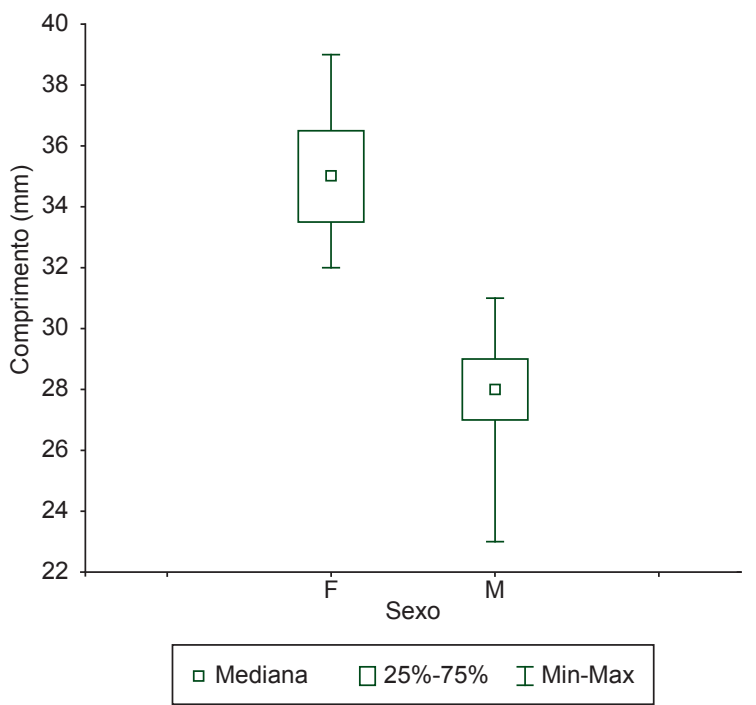

Figura 3. Valores medianos, máximos, mínimos e percentis do comprimento padrão (mm) de machos e fêmeas de Corydoras flaveolus.

Figure 3. Median values, minimum, maximum and percentiles for the standard length (mm) of males and females of Corydoras flaveolus.

$(\mathrm{U}=6,87 ; \mathrm{p}<0,001)$ entre os comprimentos de machos e fêmeas, sendo as fêmeas maiores do que os machos.

As freqüências dos diferentes graus de repleção e gordura acumulada na cavidade visceral nas épocas de seca e cheia encontram-se na Figura 4. Não houve associação entre os diferentes graus de repleção $\left(\chi^{2}=0,06 ; \mathrm{gl}=2 ; \mathrm{p}=0,212\right)$ e de gordura acumulada na cavidade visceral $\left(\chi^{2}=2,52 ; \mathrm{gl}=2 ; \mathrm{p}=0,284\right)$ com a época do ano.

Dos 70 indivíduos capturados, 1,35\% apresentaram gônadas imaturas (estádio A), 20,27 \% em maturação ou repouso (estádio B) e $78,38 \%$ maduras (estádio C). Considerando cada amostragem, os indivíduos maduros estiveram presentes a partir do mês de julho, sendo a maior porcentagem encontrada no mês de novembro (Figura 5).

A relação gonadossomática média calculada para o período de seca (de maio a setembro) foi 8,49 e para o período de cheia (de novembro a março) 10,63 . Com isso, pode-se dizer que o período reprodutivo de Corydoras flaveolus é extenso, sendo mais intenso na cheia, visto a maior porcentagem de indivíduos com gônadas maduras e o maior valor da relação gonadossomática média durante esse período.

Foram analisados 30 pares de ovários maduros de $C$. flaveolus. A distribuição de freqüência dos diâmetros de ovócitos para cada um desses pares está apresentada na Figura 6. Os diâmetros dos ovócitos vitelogênicos variaram de 5 d. o. m. (306 $\mu \mathrm{m})$ a 39 d. o. m. $(2386,9 \mu \mathrm{m})$. A partir de 25 d. o. m. $(1530 \mu \mathrm{m})$, os lotes de ovócitos
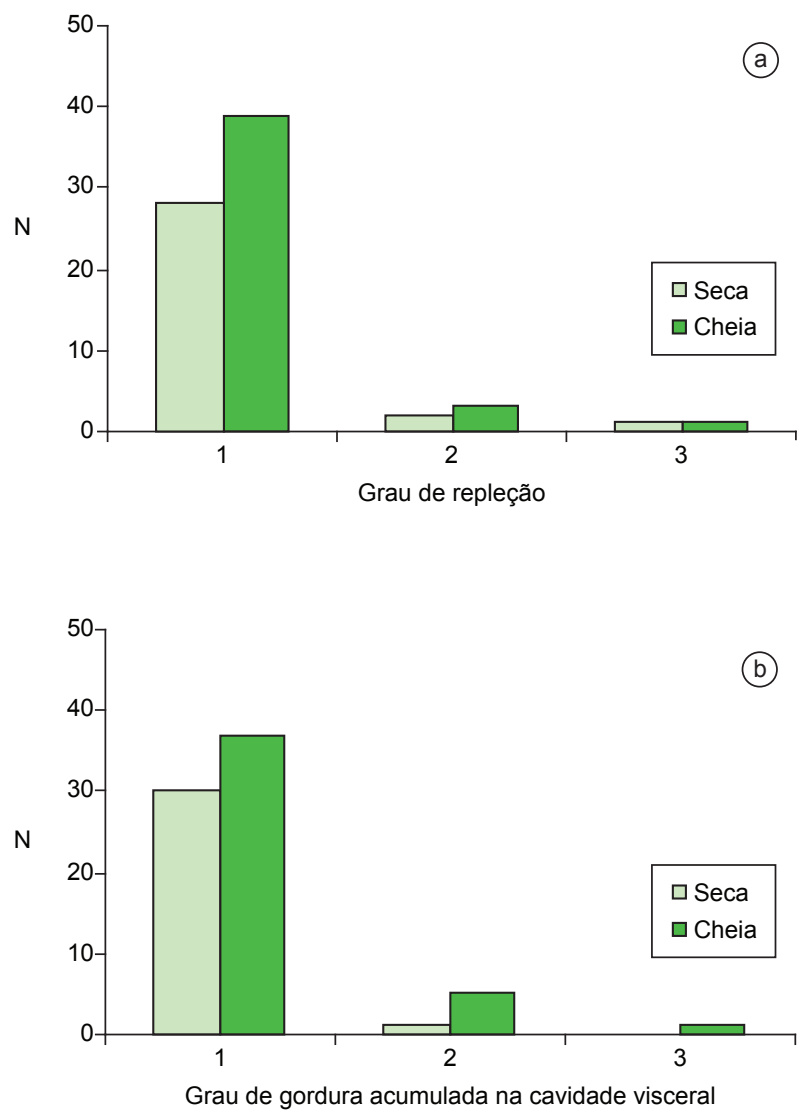

Figura 4. Distribuição numérica dos graus de repleção (a) (1 para estômagos considerados vazios, 2 para estômagos parcialmente cheios e 3 para estômagos repletos) e de gordura acumulada na cavidade visceral (b) (1 para cavidade visceral sem gordura, 2 para cavidade visceral parcialmente preenchida com gordura e 3 para cavidade visceral repleta de gordura) de Corydoras flaveolus entre os períodos de seca e cheia.

Figure 4. Numeric distribution of variations in repletion degree (a) (1 for stomachs considered empty, 2 for stomachs partially full and 3 for stomachs completely full) and fat accumulated in visceral cavity (b) (1 for the visceral cavity that doesn't present fat, 2 for the visceral cavity partially filled with fat and 3 for the visceral cavity full of fat ) of Corydoras flaveolus between drought and rainy season periods.

mais desenvolvidos estão pouco representados ou nem aparecem em alguns ovários. A eliminação de lotes de ovócitos mais desenvolvidos pode indicar um processo de desova intermitente, que é um caso extremo de desova parcelada.

A fecundidade absoluta média foi de 137 ovócitos (com \pm 51 ovócitos de desvio padrão). As fecundidades absolutas das fêmeas 


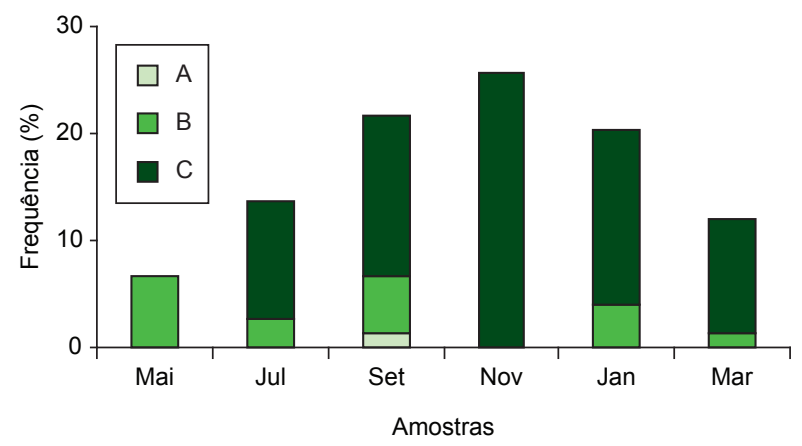

Figura 5. Frequiência dos indivíduos de Corydoras flaveolus nos diferentes estádios de maturação gonadal em cada amostragem (A: indivíduos imaturos; B: indivíduos com gônadas em maturação ou repouso; e C: indivíduos com gônadas maduras).

Figure 5. Relative frequency of Corydoras flaveolus individuals in different states of gonadal development in each sampling (A: immature individuals; B: individuals with gonads in development or rest; C: individuals with ripe gonads).
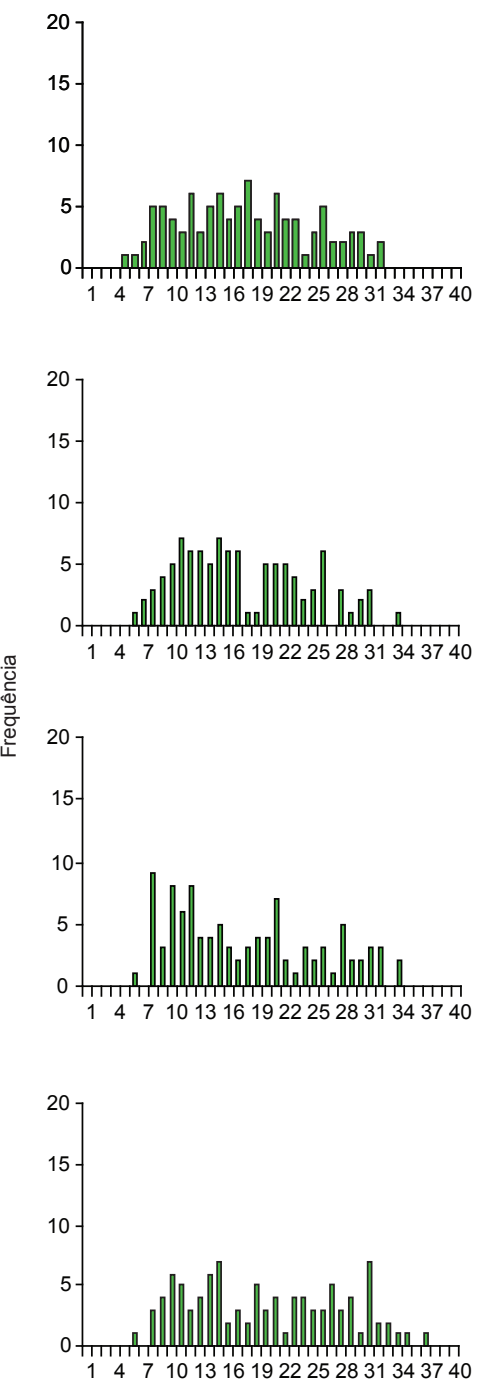
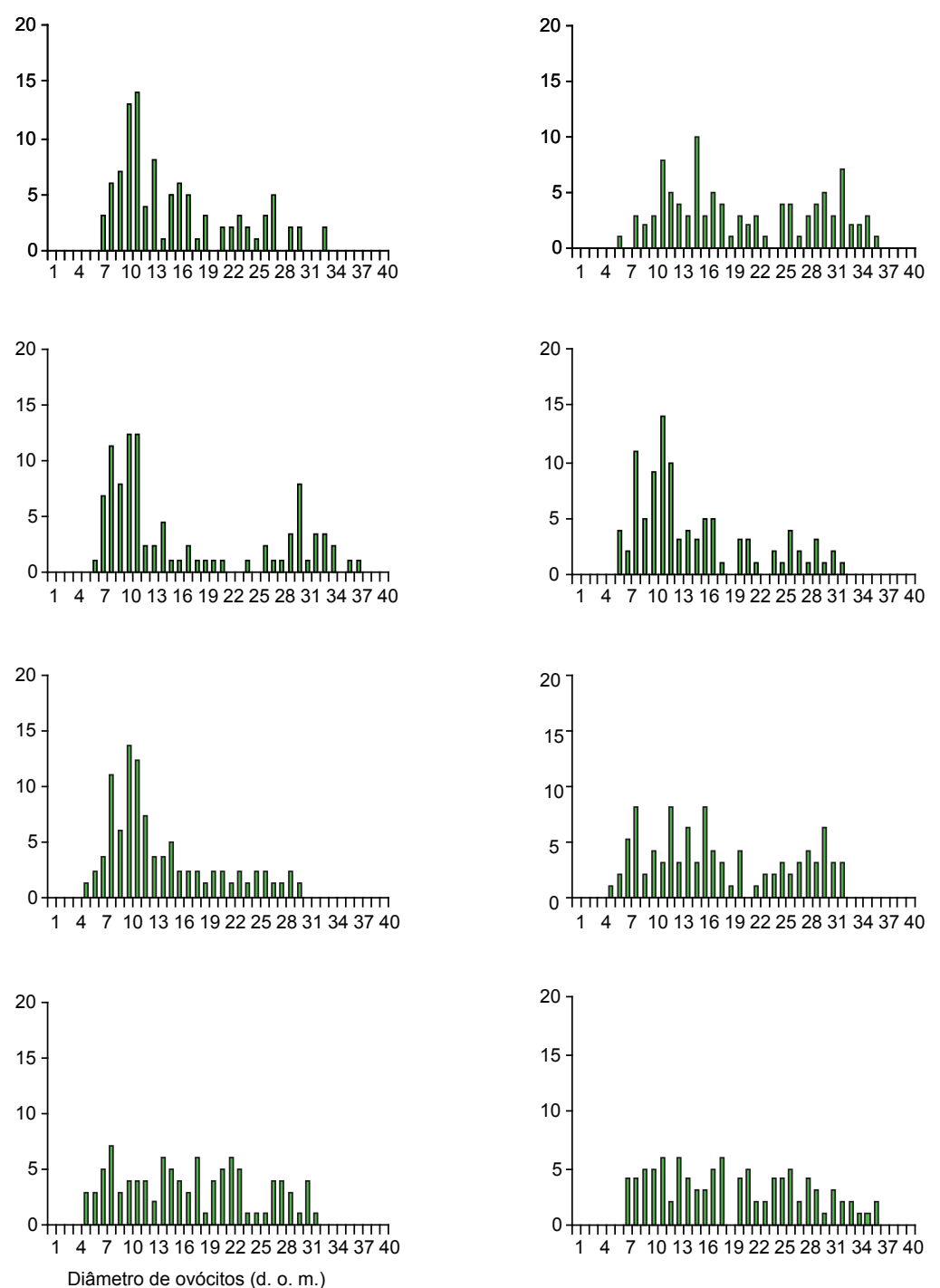

apresentaram correlação com os respectivos pesos totais, sendo a equação $\mathrm{y}=61,225 \mathrm{x}^{1,3404}(\mathrm{r}=0,76 ; \mathrm{p}<0,05)$ descritora da fecundidade da população.

Os valores estimados do intercepto, coeficiente angular e coeficiente de correlação para as relações peso e comprimento para machos e fêmeas, para todo o período de amostragem, estão apresentados na Tabela 2. As relações peso e comprimento foram diferentes para machos e fêmeas $(\mathrm{t}=$ $5,89 ; \mathrm{gl}=68 ; \mathrm{p}<0,05)$. Através dos valores dos interceptos, tem-se que as fêmeas foram relativamente mais pesadas do que os machos.

Não houve diferença significativa na condição corporal de machos e fêmeas entre as épocas de seca e cheia ( $p>0,05)$, sendo a interação comprimento-época também não significativa para ambos os sexos. As Tabelas 3 e 4 mostram os resultados da análise de variância para o peso de machos e fêmeas de $C$. flaveolus considerando a época e o comprimento padrão.

\section{Discussão}

A ocorrência de Corydoras flaveolus no Rio Passa Cinco esteve relacionada a ambientes rasos, de pouca correnteza e com vegetação

Figura 6. Distribuição de freqüência dos diâmetros de ovócitos de Corydoras flaveolus $(1$ d. o. m. $=61,2 \mu \mathrm{m})$ analisados em ovários maduros, onde se observam diferentes grupos de ovócitos em desenvolvimento, associados a uma desova intermitente.

Figure 6. Frequency distribution of oocytes diameter of Corydoras flaveolus analyzed in mature ovaries $(1 \mathrm{~d} . \mathrm{o} . \mathrm{m} .=61.2 \mu \mathrm{m})$, where there are different groups of developing oocytes, associeted with a intermittent spawning. 

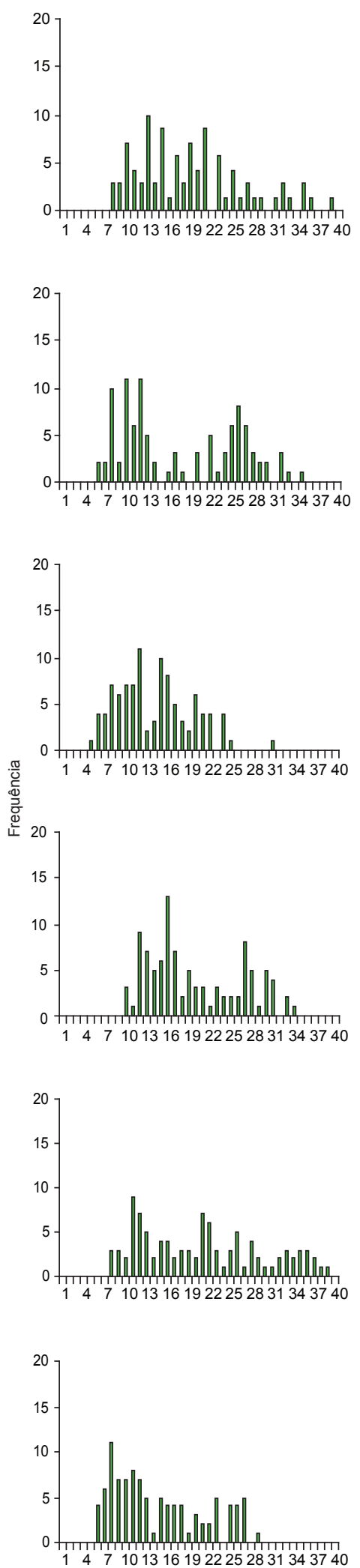
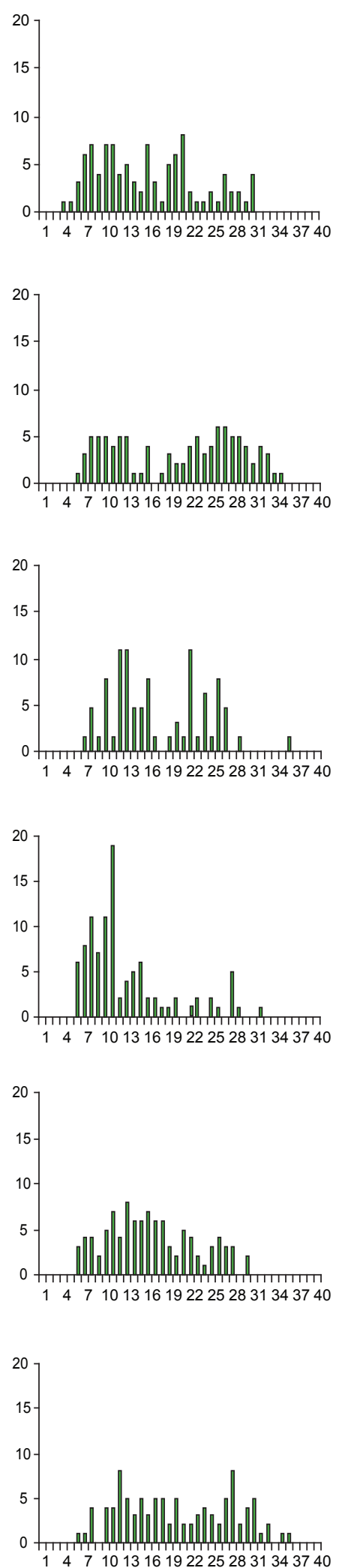

Diâmetro de ovócitos (d. o. m.)


Figura 6 (continuação). Distribuição de freqüência dos diâmetros de ovócitos de Corydoras flaveolus (1 d. o. m. = 61,2 $\mu$ m) analisados em ovários maduros, onde se observam diferentes grupos de ovócitos em desenvolvimento, associados a uma desova intermitente.

Figure 6 (continued). Frequency distribution of oocytes diameter of Corydoras flaveolus analyzed in mature ovaries $(1 \mathrm{~d}$. o. $\mathrm{m} .=61.2 \mu \mathrm{m})$, where there are different groups of developing oocytes, associeted with a intermittent spawning. 
Tabela 2. Valores estimados de a (intercepto), b (coeficiente angular) e r (coeficiente de correlação) para as relações peso e comprimento de machos e fêmeas ( $\mathrm{N}=$ número de indivíduos analisados).

Table 2. Estimated values of a (intercept), b (angular coefficient) and $r$ (correlation coefficient) in length-weight relationship of males and females, considering $\mathrm{N}$ the number of specimens analyzed.

\begin{tabular}{ccccc}
\hline Sexo & $\mathbf{a}$ & $\mathbf{b}$ & $\mathbf{r}$ & $\mathbf{N}$ \\
\hline Machos & 0,0002 & 2,46 & 0,79 & 35 \\
Fêmeas & 0,0004 & 2,34 & 0,72 & 35 \\
\hline
\end{tabular}

Tabela 3. Resultados da análise de variância para o peso de machos de Corydoras flaveolus considerando a época e comprimento padrão (FV = fonte de variação; $\mathrm{SQ}=$ soma de quadrados; $\mathrm{GL}=$ graus de liberdade; $\mathrm{QM}=$ quadrado médio; $\mathrm{F}=$ teste $\mathrm{F}$ ).

Table 3. Results of variance analysis of males' weight of Corydoras flaveolus according to period and standard length $(\mathrm{FV}=$ source of variation; $\mathrm{SQ}=\mathrm{sum}$ of squares; GL = degrees of freedom; $\mathrm{QM}=$ square medium; $\mathrm{F}=$ test $\mathrm{F}$ ).

\begin{tabular}{lccccc}
\hline \multicolumn{1}{c}{ FV } & SQ & GL & QM & F & P \\
\hline Comprimento & 0,223 & 1 & 0,223 & 46,551 & 0,0001 \\
Época & 0,002 & 1 & 0,002 & 0,494 & 0,488 \\
Resíduo & 0,144 & 30 & 0,005 & - & - \\
\hline
\end{tabular}

Tabela 4. Resultados da análise de variância para o peso de fêmeas de Corydoras flaveolus considerando a época e comprimento padrão (FV = fonte de variação; $\mathrm{SQ}=$ soma de quadrados; $\mathrm{GL}=$ graus de liberdade; $\mathrm{QM}=$ quadrado médio; $\mathrm{F}=$ teste $\mathrm{F}$ ).

Table 4. Results of variance analysis of females' weight of Corydoras flaveolus according to period and standard length ( FV = source of variation; $\mathrm{SQ}=$ sum of squares; $\mathrm{GL}=$ degrees of freedom; $\mathrm{QM}=$ square medium; $\mathrm{F}=$ test $\mathrm{F}$ ).

\begin{tabular}{lccccc}
\hline \multicolumn{1}{c}{ FV } & SQ & GL & QM & F & P \\
\hline Comprimento & 0,071 & 1 & 0,071 & 6,790 & 0,015 \\
Época & 0,016 & 1 & 0,016 & 1,527 & 0,227 \\
Resíduo & 0,294 & 28 & 0,01 & - & - \\
\hline
\end{tabular}

marginal presente. Ocupações espaciais semelhantes foram encontradas para Corydoras carlae Nijssen \& Isbrucker, 1983 e Corydoras aeneus (Gill, 1858) (Aranha et al. 1993) e Aspidoras fuscoguttatus Nijssen \& Isbrucker, 1976 (Araújo \& Garutti 2003).

A proporção sexual encontrada para $C$. flaveolus não diferiu do esperado, ocorrendo uma fêmea para cada macho. Para outras espécies de Corydoras esse padrão não foi mantido. Bervian \& Fontoura (1994) encontraram uma maior proporção de machos em Corydoras paleatus (Jenyns, 1842), assim como Aranha et al. (1993) para C. aeneus. A maior proporção de fêmeas ocorreram nos estudos de Aranha et al. (1993) com Corydoras carlae Nijssen \& Isbrücker, 1983 (66\%) e Alkins-Koo (2000) com C. aeneus. De acordo com Nikolsky (1963), a proporção entre os sexos varia consideravelmente de espécie para espécie, podendo também variar na mesma população de um ano para o outro.

As fêmeas de C. flaveolus foram maiores que os machos. Resultados semelhantes foram encontrados para outras espécies do gênero (Pruzsinsky \& Ladich 1998, Alkins-Koo 2000, Araújo \& Garutti 2002, Kohda et al. 2002, Mazzoldi et al. 2007). Bervian \& Fontoura (1994), através da análise da estrutura etária de $C$. paleatus, verificaram que as fêmeas, além de atingirem comprimentos ligeiramente superiores aos dos machos, apresentaram maiores taxas de crescimento.
Nikolsky (1963) e Lowe-McConnell (1999) mostram que a forma mais freqüente de dimorfismo sexual entre peixes é o maior tamanho alcançado pelas fêmeas. De acordo com Wootton (1992), há uma correlação positiva entre o tamanho da fêmea e a fecundidade, o que propicia maior fecundidade para fêmeas de maiores tamanhos. Peixes de riachos que habitam ambientes limitados espacialmente e sujeitos a variações sazonais no volume de água, desenvolvem notórias adaptações morfológicas internas (Braga 2004), de comprimentos entre machos e fêmeas (Braga 2005) e de outros atributos entre sexos como taxa de crescimento e mortalidade natural (Braga et al. 2007).

O tipo de desova que uma espécie de peixe pode apresentar é uma das inúmeras táticas que integram as estratégias reprodutivas (Vazzoler 1996). A dinâmica do desenvolvimento dos ovócitos caminha de uma eliminação total de ovócitos maduros, quando a espécie apresenta um período reprodutivo curto, para uma eliminação de ovócitos maduros em lotes sucessivos, sendo, nesse caso, um período reprodutivo mais longo (Nikolsky 1963). Nos peixes de ambientes temperados-frios ou peixes migradores (inclusive tropicais) a desova pode ser total, enquanto que nos peixes de ambientes tropicais ou sub-tropicais (onde as condições ambientais propícias são mais longas, mas há menor disponibilidade de alimento para as larvas e pós-larvas que passam a fazer uso do alimento exógeno) os ovócitos são eliminados em lotes e o período reprodutivo é mais longo (Nikolsky 1963).

Vazzoler (1996) descreve a desova em que os ovócitos são eliminados em lotes durante o período reprodutivo como "sincrônico em mais de dois grupos", o que caracteriza uma desova múltipla ou parcelada. Quando não se evidenciam períodos reprodutivos definidos, a desova é considerada intermitente. Tais considerações encontram respaldo em Wallace \& Selman (1981) e West (1990).

O período reprodutivo longo, a existência de vários lotes de ovócitos em desenvolvimento e a não ocorrência de indivíduos com gônadas esgotadas sugerem que a espécie possa apresentar uma desova intermitente, que é um caso extremo de desova parcelada (Vazzoler 1996).

Vazzoler \& Menezes (1992) relataram que o período reprodutivo para as espécies da bacia do Rio Paraná inicia-se em outubro, quando a temperatura é elevada e o nível das águas começa a subir, atingindo seu pico em dezembro-janeiro, quando o nível pluviométrico é alto. A reprodução de Corydoras flaveolus, no rio Passa Cinco, condiz com essas informações, apresentando um período reprodutivo longo, sendo mais intenso durante a estação chuvosa. Alkins-Koo (2000) relatou um período reprodutivo semelhante para C. aeneus, assim como Aranha et al. (1993) para C. carlae.

A fecundidade é uma característica específica e está adaptada às condições do ciclo de vida da espécie, variando com o crescimento, densidade populacional, disponibilidade de alimento e taxa de mortalidade (Nikolsky 1969).

Bervian \& Fontoura (1994) encontraram uma fecundidade máxima de 400 ovócitos em C. paleatus. Araújo \& Garutti (2002) verificaram que Aspidoras fuscoguttatus apresentou uma fecundidade de 51 a 166 ovócitos e uma variação de 159 a 1749 um no diâmetro dos ovócitos. Alkins-Koo (2000) encontrou uma fecundidade máxima de 1046 ovócitos para C. aeneus sendo 1,14 mm o diâmetro mínimo de ovócitos maduros. Com base nessas informações, para outras espécies da subfamília Corydoradinae, C. flaveolus apresentou uma fecundidade baixa e ovócitos bastante grandes. De acordo com Wootton (1992), a redução na fecundidade é compensada por ovócitos maiores, que dão origem a larvas maiores, com capacidade de melhor explorar o ambiente.

Assim, acredita-se que Corydoras flaveolus enquadre-se na estratégia oportunista proposta por Winemiller (1989), visto tratar-se de um peixe de pequeno porte, que apresenta maturação precoce e período 
reprodutivo prolongado. Além disso, é provável que $C$. flaveolus apresente algumas táticas comportamentais que viabilizem o sucesso da fecundação dos óvulos, como demonstrado por Kohda et al. (1995), já que a espécie apresenta desova intermitente, baixa fecundidade e ovócitos grandes.

A relação peso e comprimento é um importante parâmetro das populações de peixes e suas aplicações variam desde a estimativa do peso de um indivíduo, conhecido o seu comprimento, até indicações da condição corporal dos peixes, além de ser útil como indicadora do acúmulo de gordura e desenvolvimento das gônadas (Le Cren 1951).

De acordo com Bennemann et al. (1996), as alterações na condição corporal estão associadas às mudanças fisiológicas que ocorrem em função do ciclo reprodutivo das espécies, sendo que a atividade alimentar, o desenvolvimento de gordura e a reprodução dos peixes são dependentes do ciclo hidrológico.

Corydoras flaveolus não apresentou diferenças significativas na condição corporal de machos e fêmeas nas épocas de seca e cheia, nem na atividade alimentar e acúmulo de gordura. $\mathrm{O}$ maior peso relativo apresentado pelas fêmeas deve ser atribuído ao alto investimento na reprodução. Os ovócitos grandes e ricos em vitelo apresentados pelas fêmeas dessa espécie contribuem, e muito, para um incremento em peso.

Mazzoldi et al. (2007) investigaram a relação entre os modos de fertilização e a morfologia dos testículos de quatro espécies de Corydoras (Corydoras aeneus, Corydoras bondi Gosline, 1940, Corydoras ehrhardti Steindachner, 1910 e Corydoras potaroensis Myers, 1927) e verificaram a presença de um tipo de espermatogênese que conduz a uma redução no número de gametas, o que indica um baixo investimento em gônadas nos machos (Kohda et al. 2002). Como os espermatozóides são liberados em um ambiente restrito (no interior de uma bolsa formada pelas nadadeiras pélvicas da fêmea) (Kohda et al. 1995), não é necessária uma grande quantidade de espermatozóides. Caso C. flaveolus siga essa mesma estratégia reprodutiva, justifica-se o peso relativo inferior dos machos quando comparado às fêmeas.

\section{Agradecimentos}

A autora agradece à FAPESP pela bolsa de mestrado concedida (proc. $n^{\circ}$ 04/12669-3), ao IBAMA (proc. $n^{\circ}$ 02027.000234/2005-05) pela licença para execução dos trabalhos de campo e aos revisores anônimos pelas valiosas sugestões.

\section{Referências Bibliográficas}

ALKINS-KOO, M. 2000. Reproductive timing of fishes in a tropical intermittent stream. Environ. Biol. Fishes. 57:49-66.

ARANHA, J.M.R., CARAMASCHI, E.P. \& CARAMASCHI, U. 1993. Ocupação espacial, alimentação e época reprodutiva de duas espécies de Corydoras Lacépède (Siluroidei, Callichthyidae) coexistentes no rio Alambari (Botucatu, São Paulo). Rev. Bras. Zool. 10(3):453-466.

ARAÚJO, R.B. \& GARUTTI, V. 2002. Biologia reprodutiva de Aspidoras fuscoguttatus (Siluriformes, Callichthyidae) em riacho de cabeceira da bacia do Alto rio Paraná. Iheringia, Sér. Zool. 92(4):89-98.

ARAÚJO, R.B. \& GARUTTI, V. 2003. Ecology of a stream from Upper Paraná river basin inhabited by Aspidoras fuscoguttatus Nijssen \& Isbrucker, 1976 (Siluriformes, Callichthyidae). Braz. J. Biol. 63(3):363-372.

ARTONI, R.F., TERÊNCIO, M.L., VICAR, M.R., MATIELO, M.C.A., CESTARI, M.M. \& BERTOLLO, L.A.C. 2006. Cytogenetics of two sympatric Corydoras species (Pisces, Siluriformes, Callichthyidae) of southern Brazil. Braz. J. Biol. 66(1B):191-198.

AXENROT, T.E. \& KULlANDER, S.O. 2003. Corydoras diphyes (Siluriformes: Callichthyidae) and Otocinclus mimulus (Siluriformes:
Loricariidae), two new species of catfishes from Paraguay, a case of mimetic association. Ichthyol. Explor. Freshwaters. 14(3):249-272.

BENNEMANN, S.T., ORSI, M.L. \& SHIBATA, O.A. 1996. Atividade alimentar de espécies de peixe do rio Tibagi, relacionada com o desenvolvimento de gordura e gônadas. Rev. Bras. Zool. 13(2):501-512.

BERVIAN, G. \& FONTOURA, N.F. 1994. Dinâmica populacional da ictiofauna da lagoa Fortaleza, Cidreira, Rio Grande do Sul. III Corydoras paleatus (Jenyns, 1842) (Teleostei, Callichthyida). Biociências. 2(2):15-23.

BRAGA, F.M.S. 1990. Aspectos da reprodução e alimentação de peixes comuns em um trecho do rio Tocantins entre Imperatriz e Estreito, Estado do Maranhão e Tocantins, Brasil. Rev. Bras. Biol. 50(3):547-558.

BRAGA, F.M.S. 2004. Habitat, distribuição e aspectos adaptativos de peixes da microbacia do Ribeirão Grande, Estado de São Paulo, Brasil. Acta Sci. Biol. Sci. 26(1):31-36.

BRAGA, F.M.S. 2005. Spatial distribution of the characidiin fishes (Teleostei, Crenuchidae) in the Ribeirão Grande system, a tributary of Paraíba do Sul river. Acta Sci. Biol. Sci. 27(3):259-263.

BRAGA, F.M.S., SOUZA, U.P. \& CARMASSI, A.L. 2007. Dinâmica populacional de Characidium lauroi e C. alipioi (Teleostei, Crenuchidae) na micorbacia do Ribeirão Grande, serra da Mantiqueira Oriental, estado de São Paulo. Acta Sci. Biol. Sci. 29(3):281-287.

BRITO, M. 2003. Phylogeny of the subfamily Corydoradinae Hoedeman, 1952 (Siluriformes: Callichthyidae), with a definition of its genera. Proc. Acad. Nat. Sci. Phila. 153:119-154.

BRITTO, M.R. \& CASTRO, R.M.C. 2002. New corydoradine catfish (Siluriformes: Callichthyidae) from the Upper Paraná and São Francisco: the sister group of Brochis and most of Corydoras species. Copeia. (4):1006-1015.

BRITTO, M.R. \& LIMA, F.C.T. 2003. Corydoras tukano, a new species of corydoradine catfish from the rio Tiquié, upper rio Negro basin, Brazil (Ostariophysi: Siluriformes: Callichthyidae). Neotrop. Ichthyol. 1(2):83-91.

BRITTO, M.R., LIMA, F.C.T. \& HIDALGO, M.H. 2007. Corydoras ortegai, a new species of corydoradine catfish from the lower rio Putumayo in Peru (Ostariophysi: Siluriformes: Callichthyidae). Neotrop. Ichthyol. 5(3):293-300.

CETRA, M. 2005. Avaliação do bem estar em peixes: ANCOVA uma alternativa para estudos da relação peso-comprimento. Bol. Soc. Bras. Ictiol. (81):3-5.

FOWLER, J., COHEN, L. \& JARVIS, P. 1998. Practical statistics for field biology. John Wiley \& Sons, England.

GARCIA, G.J., HEBERT, H.D., PERINOTTO, J.A.J. \& CASTRO, J.F.M. 2004. Atlas ambiental da bacia do rio Corumbataí.http://ceapla.rc.unesp. br/atlas/ (último acesso em 13/03/2008).

HUYSENTRUYT, F. \& ADRIAENS, D. 2005. Adhesive structures in the eggs of Corydoras aeneus (Gill, 1858; Callichthyidae). J. Fish Biol. 66:871-876.

KAATZ, I.M. \& LOBEL, P.S. 1999. Acoustic behaviour and reproduction in five species of Corydoras catfishes (Callichthyidae). Biol. Bull. 197:241-242.

KOHDA, M., TANIMURA, M., KIKUE-NAKAMURA, M. \& YAMAGISHI, S. 1995. Sperm drinking by female catfishes: a novel mode of insemination. Environ. Biol. Fishes. 42:1-6.

KOHDA, M., YONEBAYASHI, K., NAKAMURA, M., OHNISHI, N., SEKI, S., TAKAHASHI, D. \& TAKEYAMA, T. 2002. Male reproductive success in a promiscuous armoured catfish Corydoras aeneus (Callichthyidae). Environ. Biol. Fishes. 63:281-287.

LE-CREN, E.D. 1951. The lenght-weight relationship and seasonal cycle in gonad weight and condition in the perch (Perca fluviatilis). J. Anim. Ecol. 20(2):201-219.

LOIR, M., CAUTY, C., PLANQUETE, P. \& BAIL, P.Y.L. 1989. Comparative study of the male reproductive tract in seven families of South-American catfishes. Aquatic Living Resour. 2:45-56. 
LOWE-MCCONNELL, R.H. 1999. Estudos ecológicos em comunidades de peixes tropicais. Editora USP, São Paulo.

MAZZOLDI, C., LORENZI, V. \& RASOTTO, M.B. 2007. Variation of male reproductive apparatus in relation to fertilization modalities in the catfish families Auchenipteridae and Callichthyidae) (Teleostei: Siluriformes). J. Fish Biol. 70:243-256.

NIKOLSKY, G.V. 1963. The ecology of fishes. Academic Press, London.

NIKOLSKY, G.V. 1969. Theory of fish population dynamics. Oliver \& Boyd, Edinburgh.

PAXTON, C.G.M. 1997. Shoaling and activity levels in Corydoras. J. Fish Biol. 51:496-502.

PRUZSINSZKY, I. \& LADICH, F. 1998. Sound production and reproductive behaviour of the armoured catfish Corydoras paleatus (Callichthyidae). Environ. Biol. Fishes. 53:183-191.

REIS, R.E. 2003. Family Callichthyidae (armored catfishes). In Check list of the freshwater fishes of South and Central America (R.E Reis, S.O. Kullander \& C.J. Ferraris, eds.). EDIPUCRS, Porto Alegre.

SHIBATTA, O.A. \& HOFFMANN, A.C. 2005. Variação geográfica em Corydoras paleatus (Jenyns) (Siluriformes, Callichthyidae) do sul do Brasil. Rev. Bras. Zool. 22(2):366-371.

SHIMABUKURO-DIAS, C.K., OLIVEIRA, C. \& FORESTI, F. 2004 Cytogenetic analysis of five species of the subfamily Corydoradinae (Teleostei: Siluriformes: Callichthyidae). Genet. Molecular Biol. 27(4):549-554.

SOKAL, R.R. \& ROHLF, F.J. 1995. Biometry. WH Freeman and Company, New York.

SPADELLA, M.A., OLIVEIRA, C. \& QUAGIO-GRASSIOTTO, I. 2007. Comparative analysis of spermiogenesis and sperm ultastructue in Callichthyidae (Teleostei: Ostariophysi: Siluriformes). Neotrop. Ichthyol. 5(3):337-350.
SPARRE, P. \& VENEMA, S.C. 1997. Introdução à avaliação de mananciais de peixes tropicais.

FAO, Roma. (Documento técnico sobre as pescas. Parte 1: Manual).

STRAHLER, A.L. 1952. Dynamic baisis of geomorphology. Geol. Soc. Am. Bull. 63:923-938.

STRAUSS, R.E. 1985. Evolutionary allometry and variation in body form in the south american catfish genus Corydoras (Callichthyidae). Syst. Zool. 34(4):381-396

VALENTE, R.O.A. \& VETTORAZZI, C.A. 2002. Análise da estrutura da paisagem na bacia do rio Corumbataí, SP. Scientia Forestalis. 62:114 129.

VAZZOLER, A.E.A.M. \& MENEZES, N.A. 1992. Síntese de conhecimento sobre comportamento reprodutivo de Characiformes da América do Sul (Teleostei, Ostariophysi). Rev. Bras. Biol. 52(4):627-640.

VAZZOLER, A.E.A.M. 1996. Biologia da reprodução de peixes teleósteos: teoria e prática. EDUEM; SBI, São Paulo.

VIEIRA, S. 2004. Bioestatística: tópicos avançados. Elservier, Rio de Janeiro.

WALLACE, R.A. \& SELAMN, K. 1981. Cellular and dynamic aspects of oocyte growth in teleosts. Amer. Zool. 21:325-343.

WEST, G. 1990. Methods of assessing ovarian development in fishes: a review. Aust. J. Mar. Freshwater Res. 41:199-222.

WINEMILLER, K.O. 1989. Patterns of variation in life history among South American fishes in seasonal environments. Oecologia. 81:225-241.

WOOTTON, R.J. 1992. Fish ecology. Chapman \& Hall, New York.

Recebido em 09/04/09

Versão reformulada recebida em 03/06/09

Publicado em 15/10/09 